\title{
ESTRUCTURA Y COMPOSICIÓN VEGETAL DE UN BOSQUE SECO TROPICAL EN REGENERACIÓN EN BATACLÁN (CALI, COLOMBIA)
}

\section{Vegetation structure and composition of a tropical dry forest in regeneration in Bataclán (Cali, Colombia)}

\author{
Viviana Londoño Lemos \& Alba Marina Torres González²
}

Londoño L, V. \& Torres G, A.M. (2015). Estructura y composición vegetal de un bosque seco tropical en regeneración en Bataclán (Cali, Colombia). Colombia Forestal, 18(1),71-85

Recepción: 28 de octubre de 2014

\section{RESUMEN}

La caracterización de la vegetación a largo plazo en los bosques secos tropicales (bs-T) en regeneración permite establecer los patrones de composición, estructura y dinámica de las comunidades vegetales, así como conocer los diferentes estados del proceso de sucesión vegetal. En este estudio se evaluó la comunidad vegetal del bosque del ecoparque Bataclán, Cali, Colombia; mediante la determinación de su estructura y composición en dos estrategias de regeneración. Una de ellas consistió en regeneración natural con barreras de bambú y la otra en regeneración natural sin barreras de bambú. Se establecieron tres parcelas permanentes de $500 \mathrm{~m}^{2}$ en cada una de las estrategias de regeneración (seis parcelas en total). Se determinó la composición y estructura, teniendo en cuenta todos los hábitos de crecimiento, con diferentes tipos de muestreo. Se encontró que no hay diferencias significativas entre la estructura y composición vegetal de las dos estrategias de regeneración del bosque. Se registraron 41 especies pertenecientes a 27 familias (árboles y arbustos $58.5 \%$, hierbas $24.4 \%$, trepadoras o escandentes $14.6 \%$, epífitas $2.4 \%$ ). La familia dominante fue
Aprobación: 9 de diciembre de 2014

Melastomataceae y la especie dominante fue Miconia prasina. La orquídea Catasetum ochraceum y el pasto Thrasya petrosa fueron especies indicadoras de alta luminosidad en el bosque. Se concluye que la comunidad vegetal se encuentra en un estado sucesional temprano, donde existe una mezcla de especies plantadas y otras naturalmente regeneradas en la zona, caracterizada por especies pioneras provenientes de bs-T cercanos y de otras zonas de vida. Palabras clave: barreras de bambú, bosques en regeneración, composición florística, estructura vegetal, parcelas permanentes, Phyllostachys aurea, sucesión.

\begin{abstract}
Characterization of long-term vegetation in a tropical dry forest (TDF) that is in the process of regeneration permits establishment of patterns of composition, structure and dynamics of plant communities and sheds light on the different stages of plant succession. In this study, the plant community of ecoparque Bataclán, Cali, Colombia was evaluated by determining its structure and composition in two regeneration strategies. One strategy consisted
\end{abstract}

1 Grupo de investigación Ecología y Diversidad Vegetal, Universidad del Valle, Cali, Colombia. vlondon28@gmail.com. Autor para correspondencia.

2 Grupo de investigación Ecología y Diversidad Vegetal, Universidad del Valle, Cali, Colombia. alba.torres@correounivalle.edu.co 
of natural regeneration with bamboo barriers and the other of natural regeneration without bamboo barriers. Three permanent plots of $500 \mathrm{~m}^{2}$ were established in each regeneration strategy (six plots in total). Composition and structure was determined, taking into account all the growth habits, with different sampling methods. We found no significant differences between vegetation structure and composition of the two strategies for forest regeneration. forty-one species belonging to 27 families were recorded (trees and shrubs $58.5 \%$, herbs $24.4 \%$, climbers or scandents $14.6 \%$, epiphytes $2.4 \%$ ). The dominant family was Melastomataceae and the dominant species was Miconia prasina. The orchid Catasetum ochraceum. and the grass Thrasya petrosa were indicator species for high luminosity. We conclude that the plant community is in an early successional stage, where there is a mixture of planted and naturally regenerated species in the zone, characterized by pioneer species from TDF and other nearby life zones.

Keywords: bamboo barriers, forest in regeneration, floristic composition, vegetation structure, permanent plots, Phyllostachys aurea, succession.

\section{INTRODUCCIÓN}

Los ecosistemas tropicales son los más biodiversos del planeta (Calle, 1994), sin embargo, durante el último siglo la biodiversidad en dichos ecosistemas se ha ido deteriorando debido a la reducción de los mismos por el avance de los procesos de urbanización e industrialización (Rudell et al., 2002). En América, esta realidad se extiende por todo el continente y en muchos casos los bosques naturales han dado paso a formaciones secundarias, que la mayoría de las veces son el resultado de tierras abandonadas después de su uso prolongado (Carim et al., 2007). Este acelerado cambio de los ecosistemas naturales genera la necesidad de estudiar lo que queda de los mismos, así como de monitorear los procesos de sucesión de los ecosistemas secundarios (Brown \& Lugo, 1990).

Colombia es uno de los países en el mundo con mayor biodiversidad (Sánchez, 2002). Para el caso de las plantas, se estima que alberga 24405 especies de las 90000 que se han estimado para Sur América (Camacho et al., 1992; Calle, 1994; Van der Hammen \& Rangel, 1997; Sánchez, 2002; Jørgensen et al. 2011). Sin embargo, los estudios de esta diversidad se han enfocado en ciertas zonas del territorio colombiano, dejando algunos ecosistemas muy poco representados en cuanto a investigación científica, como lo ha sido el bosque seco tropical (bs-T) (IAvH, 1998).
El bs-T corresponde a la formación vegetal que se encuentra entre los 0 y los $1000 \mathrm{~m}$ de altitud, con temperaturas superiores a los $24{ }^{\circ} \mathrm{C}$ y con uno o dos periodos de sequía al año (Janzen, 1988; $\mathrm{IAvH}$, 1998). En Colombia, el bs-T es el segundo ecosistema en extensión, ocupando el $24.97 \%$ de su territorio (González \& Devia, 1994). Sin embargo, también es considerado como el ecosistema más degradado y menos conocido del país, debido a que se encuentra en zonas donde ha habido un mayor desarrollo ganadero y agrícola, ya que sus tierras son apropiadas para este tipo de prácticas (Janzen, 1988; IAvH, 1998).

La caracterización de las comunidades vegetales en regeneración en el bs-T que conduce al conocimiento de la composición, estructura y dinámica de las mismas, es vital para entender cómo deben ser llevados a cabo los planes de restauración y reforestación para su regeneración (Rodríguez \& Arroyo, 2004; Uslar et al., 2004). En Colombia, hasta hace pocos años, la mayoría de los estudios en bs-T se habían reducido a inventarios florísticos, sin tener en cuenta aspectos de la estructura y dinámica de las comunidades estudiadas (González \& Devia, 1994), enfocándose en su mayoría en la región Caribe y valle del río Magdalena, dejando atrás otras zonas de bs-T como el valle del río Cauca $(\mathrm{IAvH}, 1998$; Torres et al., 2012a; Vargas, 2012). 
La importancia de los ecosistemas secos radica en su diversidad, que refleja una gran variedad de adaptaciones e interacciones de las plantas para afrontar el déficit hídrico y las altas temperaturas (Uslar et al., 2004; Adarve et al., 2010). Otro factor que resulta de importancia actualmente es que es el ecosistema que está siendo fraccionado a una tasa más alta en Colombia (IAvH, 1998). Por todo esto surge la necesidad de conocer qué tipos de estrategias pueden ser implementadas en las zonas de bs-T que se vayan a regenerar, que puedan llegar a ser útiles para procurar la misma. Por esta razón, el objetivo principal de esta investigación fue comparar la estructura y composición florística en dos estrategias de regeneración en el bs-T del ecoparque Bataclán de Cali, Valle del Cauca.

\section{MATERIALES Y MÉTODOS}

\section{Área de estudio}

El ecoparque Bataclán se ubica en el costado oriental del Cerro de las Tres Cruces a $3^{\circ} 27^{\prime} 39^{\prime \prime} \mathrm{N}$; $76^{\circ} 32^{\prime} 24.6^{\prime \prime} \mathrm{W}$, entre los 1000 y los $1200 \mathrm{~m}$ de altitud, en la parte alta del barrio Juanambú, comuna 2 de la ciudad de Cali, Colombia. Esta área tiene 18 ha, es protegida por el Departamento Administrativo de Gestión del Medio Ambiente (DAGMA), de Cali; cuenta con una temperatura promedio de 25 ${ }^{\circ} \mathrm{C}$ y una precipitación promedio de $909 \mathrm{~mm}$ al año (IDEAM, 2005; DAGMA, 2010). Dadas las características climáticas y de altitud se clasifica según el sistema de zonas de vida de Holdridge (1967) como bosque seco tropical (bs-T), teniendo algunas zonas de transición a bosque seco premontano (bsPM), que es el tipo de bosque que se encuentra en la parte alta del Cerro de las Tres Cruces, zona que no hace parte del ecoparque. Durante muchos años el área fue ocupada por familias de mineros que extraían carbón en la zona con métodos artesanales, lo que ha producido problemas de erosión en los predios del ecoparque Bataclán (Velásquez \& Meyer, 1994; DAGMA, 2010). Esta zona ha presentado un régimen de degradación desde la época de la colonia (P. Silverstone-Sopkin com. pers.).

Con el objetivo de solucionar los problemas de erosión que afronta el ecoparque Bataclán, el DAGMA desarrolló dos tipos de estrategias de regeneración que fueron emprendidas hace aproximadamente veinte años (L. A. Forero, com. pers.). La primera estrategia consiste en la regeneración natural con barreras de bambú chino (Phyllostachys aurea Rivière \& C.Rivière) (BB) las cuales fueron sembradas rodeando los parches de bosque; las barreras tenían una altura aproximada de $4 \mathrm{~m}$ y un ancho de cobertura de 2 a $4 \mathrm{~m}$. Esta especie de bambú es considerada controladora de la erosión debido a su rizoma leptomorfo y yemas que pueden producir tallos o rizomas indistintamente, característica que lo hace altamente invasivo, a menos que se realice un adecuado manejo de las barreras sembradas (Londoño, 2004). Para la otra estrategia se permitió la regeneración natural del bosque sin ninguna barrera $(\mathrm{RN})$.

\section{Establecimiento de parcelas permanentes}

En cada estrategia de regeneración (BB y RN) se establecieron tres parcelas permanentes de $20 \mathrm{~m}$ $\times 25 \mathrm{~m}$ (500 $\mathrm{m}^{2}$ cada una), para un total de seis parcelas $\left(3000 \mathrm{~m}^{2}\right)$ (Torres et al. 2012b). La ubicación de cada parcela se hizo de acuerdo con la disponibilidad de espacio en cada zona evaluada, situando el lado más largo de la parcela paralelo a las líneas de nivel de la montaña y el más corto en el sentido en que aumenta la pendiente (Torres et al. 2012b).

\section{Muestreo e identificación taxonómica de la vegetación}

En cada parcela se censaron individuos de plantas vasculares de todos los hábitos o formas de crecimiento que se encontraron en el área, teniendo en cuenta las clasificaciones por hábitos de crecimiento hechas por Font-Quer (1953) y Vallejo et al. (2005) (árboles y arbustos, hierbas, trepadoras 
o escandentes y epífitas). Para los individuos leñosos, se midieron todos los tallos de los árboles y arbustos con un diámetro a la altura del pecho a 1.3 $\mathrm{m}$ desde el suelo (DAP) mayor o igual a $2.5 \mathrm{~cm}$, su DAP, la altura del fuste y la altura total. En el caso de las hierbas, se realizó un recorrido en forma de $\mathrm{N}$ iniciando en el primer vértice de cada parcela, ubicando un cuadrante de $1 \mathrm{~m}^{2}$ cada $10 \mathrm{~m}$, para un total de ocho cuadrantes $\left(8 \mathrm{~m}^{2}\right)$ por parcela. En el centro de cada cuadrante se registró la cobertura del dosel dada por el porcentaje de luminosidad, medida con un densiómetro esférico (tipo A ${ }^{\circledR}$ ) y la cobertura del suelo por herbáceas, medida en porcentaje. Finalmente, para el muestreo de trepadoras o escandentes y epífitas vasculares, en cada parcela se realizó un recorrido sobre la diagonal de la misma, desde el primer vértice instalado. Se muestrearon todas las plantas trepadoras y epífitas vasculares que crecían sobre los árboles y arbustos presentes en la diagonal.

Se determinó el nombre científico y familia de cada individuo muestreado. Se colectaron muestras de herbario de las diferentes especies para hacer su identificación taxonómica. Posteriormente, las muestras fértiles se procesaron y depositaron en el herbario CUVC de la Universidad del Valle, con el objetivo de tener una colección de referencia de especies de las parcelas (Anexo A).

\section{Análisis de datos}

Para cada parcela de las dos estrategias de regeneración se realizó una curva de acumulación de especies teniendo en cuenta los estimadores de Jacknife y Bootstrap en el programa EstimateS 9.1 (Colwell, 2013). Asimismo, para cada hábito de crecimiento se calcularon los índices de diversidad de Shannon $\left(\mathrm{H}^{\prime}\right)$ y de Simpson (1-D) de cada parcela y se compararon los índices obtenidos entre estrategias de regeneración. Finalmente, se realizó un análisis de similitud (ANOSIM) de una vía con el índice de Bray-Curtis para establecer la existencia de diferencias en la composición de especies entre las dos estrategias de regeneración.
Para las especies leñosas se calculó el índice de valor de importancia (IVI) por especie y por familia y el área basal para cada parcela de cada estrategia de regeneración (Rangel \& Velásquez, 1997). Para establecer la existencia de diferencias entre áreas basales de las parcelas de cada estrategia de regeneración se realizó la prueba de Mann Whitney. Tanto para el cálculo de índice de diversidad, como el análisis de similitud y las pruebas de Mann Whitney, se utilizó el programa PAST 2.17c (Hammer et al., 2001).

Se utilizó el coeficiente de correlación de Spearman con el programa PC-ORD 5 (McCune \& Mefford, 2006) con el propósito de establecer si existía relación entre el porcentaje de cobertura de las especies de hierbas y el porcentaje de luminosidad en las parcelas. Finalmente, se estableció si existían especies de hierbas indicadoras de luminosidad (o sitios abiertos) en el bosque, mediante un análisis de especies indicadoras con el programa PC-ORD 5 (McCune \& Mefford, 2006). Para este análisis, la luminosidad se dividió en los siguientes rangos de acuerdo con los datos obtenidos en las diferentes mediciones: baja luminosidad ( $<20 \%$ ), luminosidad media ( 21 $\%>X<40 \%$ ), y alta luminosidad ( $>41 \%)$.

\section{RESULTADOS}

En las tres parcelas de la estrategia BB se registraron 642 tallos de 21 especies de árboles y arbustos pertenecientes a doce familias. Para esta zona la eficiencia de muestreo fue del $82.7 \%$ y del $92.8 \%$ según los estimadores de Jacknife y Bootstrap, respectivamente. En cuanto a las especies de hierbas para esta estrategia, se registraron ocho especies agrupadas en cinco familias. Para el hábito de trepadoras o escandentes se registraron seis especies pertenecientes a cinco familias (Tabla 1, Anexo A y Anexo B). En las parcelas de la estrategia BB no se encontraron especies epífitas.

En las tres parcelas instaladas en las zonas de la estrategia RN se registraron 761 tallos de árboles y 
arbustos de quince especies pertenecientes a nueve familias. Para esta zona la eficiencia de muestreo fue de $72.8 \%$ y $86.7 \%$ para los estimadores de Jacknife y Bootstrap, respectivamente. Se registraron seis especies del hábito trepador o escandente pertenecientes a cuatro familias botánicas. Además se registraron un total de diez especies de hierbas, pertenecientes a siete familias. Por último, se registró solo una especie de epífita, la orquídea Epidendrum melinanthum Schltr, para esta estrategia de regeneración (Tabla 1, Anexos A y Anexo B).

Los índices de diversidad para los diferentes tipos de crecimiento en las dos estrategias de regeneración del bosque Bataclán se comportaron de forma similar. No hay diferencias significativas entre la diversidad y la riqueza de especies de árboles y arbustos, hierbas, trepadoras y epífitas de las dos estrategias de regeneración (BB y RN), según la prueba de Mann Whitney (Tabla 1). Sin embargo, los valores de diversidad y riqueza de árboles y arbustos son mayores en las parcelas de la estrategia BB. Mientras que para las parcelas de la estrategia RN los valores de diversidad y riqueza son mayores en las hierbas, trepadoras y epífitas (Tabla 1). En ambas estrategias de regeneración se encontraron especies que presentaban más de una forma de crecimiento, es decir que se podían presentar como hierbas o como trepadoras o escandentes (Anexo A).
Las zonas donde se implementaron las dos estrategias de regeneración son muy similares en cuanto a composición de especies en cada uno de los hábitos de crecimiento, de acuerdo con el análisis de similitud (ANOSIM). Para árboles y arbustos $p=0.4013$, para hierbas $p=0.2022$ y para trepadoras y epífitas $p=0.6953$.

Para ambas estrategias de regeneración, las cinco familias que presentaron mayores IVI fueron Melastomataceae, Anacardiaceae, Myrtaceae, Rutaceae y Clusiaceae (Figura 1). En ambas estrategias la familia con mayor IVI fue Melastomataceae y las cuatro familias restantes varían en el valor de importancia en cada estrategia de regeneración. Así, Anacardiaceae fue la segunda en cuanto a valor de importancia para BB, mientras que Clusiaceae lo fue para $\mathrm{RN}$.

Para la estrategia de regeneración BB las especies con mayor IVI fueron Miconia prasina (Sw.) DC. (87.3\%), Mangifera indica L. (40.2\%), Zanthoxylum rhoifolium Lam. (34.3\%), Eugenia biflora (L.) DC. (32.3\%) y Clusia minor L. (22.3\%) (Figura 2). En la estrategia $\mathrm{RN}$, el mayor IVI lo obtuvo igualmente la especie $M$. prasina con un valor del $83.8 \%$, seguida por las especies Miconia rubiginosa (Bonpl.) DC. (62.0\%), C. minor (56.5\%), E. biflora (28.3\%) y M. indica (21.1\%) (Figura 2$)$. Ambas estrategias de regeneración comparten cuatro de las cinco especies dominantes (Anexo B).

Tabla 1. Riqueza y diversidad de especies para dos estrategias de regeneración del bs-T del ecoparque Bataclán, Cali, Valle del Cauca, Colombia.

\begin{tabular}{|c|c|c|c|c|c|c|c|}
\hline & \multicolumn{2}{|c|}{ Árboles y arbustos } & \multicolumn{2}{|c|}{ Hierbas } & \multicolumn{2}{|c|}{$\begin{array}{c}\text { Trepadoras y epífitas } \\
\text { vasculares }\end{array}$} & \multirow{2}{*}{$\mathbf{p}^{* * *}$} \\
\hline & BB* & $\mathbf{R} \mathbf{N}^{* *}$ & BB & RN & BB & RN & \\
\hline No. Familias & 12 & 9 & 5 & 7 & 5 & 5 & - \\
\hline No. Géneros & 18 & 12 & 8 & 10 & 6 & 7 & - \\
\hline No. Especies & 21 & 15 & 8 & 10 & 6 & 7 & 1 \\
\hline Diversidad $\left(\mathrm{H}^{\prime}\right)$ & 1.81 & 1.55 & 0.96 & 1.47 & 1.67 & 1.75 & 1 \\
\hline Diversidad (1-D) & 0.71 & 0.74 & 0.43 & 0.70 & 0.79 & 0.80 & 0.6625 \\
\hline
\end{tabular}

* BB: Regeneración natural con barreras de bambú

** RN: Regeneración natural sin barreras de bambú

*** Valores de la prueba de Mann Whitney. 


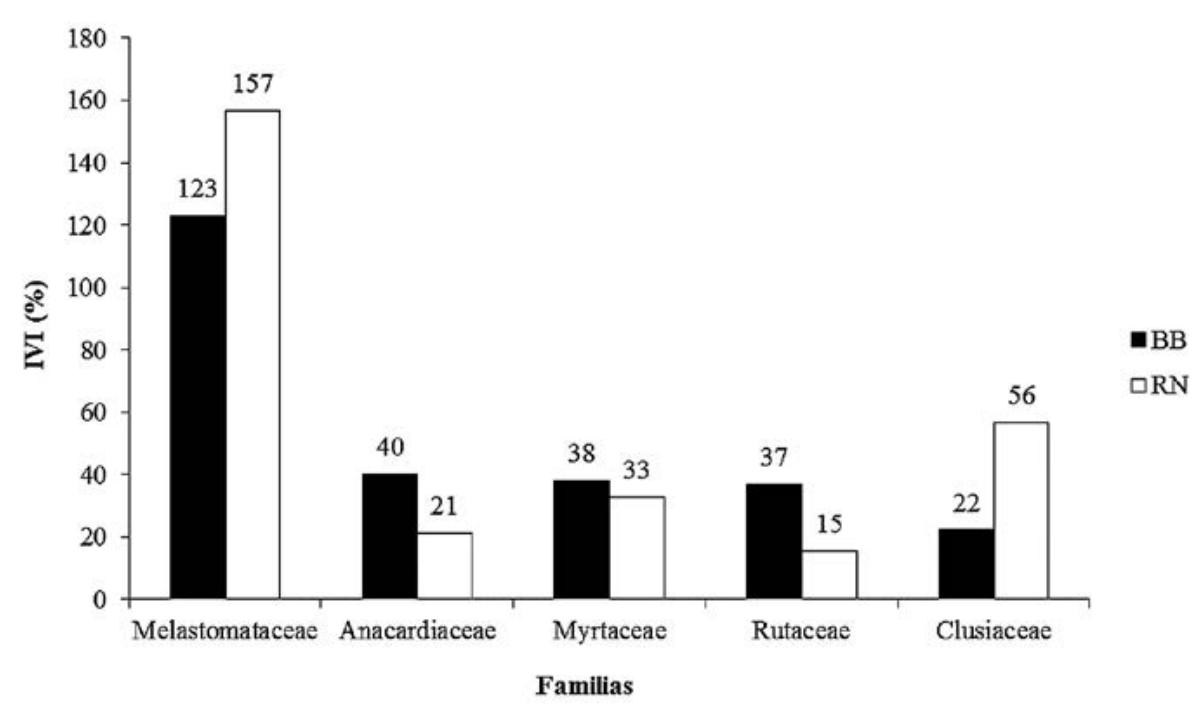

Figura 1. Familias botánicas con mayor índice de valor de importancia (IVI) en dos estrategias de regeneración del bs-T del ecoparque Bataclán, Cali, Valle del Cauca, Colombia.

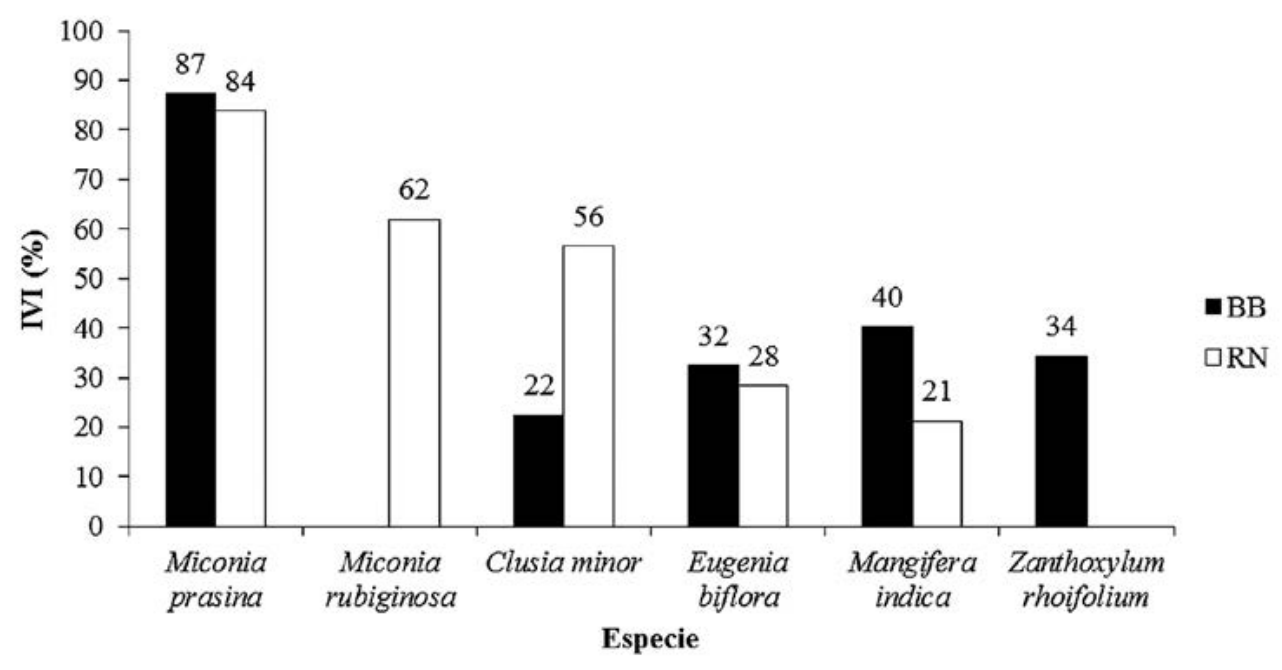

Figura 2. Especies leñosas con mayor índice de valor de importancia (IVI) en dos estrategias de regeneración del bs-T del ecoparque Bataclán, Cali, Valle del Cauca, Colombia.

El área basal presentó un comportamiento similar en las dos estrategias de regeneración del bosque. El área basal total para la estrategia BB fue de $9.2 \mathrm{~m}^{2}$ ha $^{-1}$ y de $9.8 \mathrm{~m}^{2}$ ha $^{-1}$ para la estrategia RN (Anexo B).

Se establecieron diez clases diamétricas y diez clases de altura en la vegetación arbustiva para ambas estrategias de regeneración. La estructura diamétrica de los tallos en las zonas de ambas estrategias de regeneración presentó una distribución similar, con la mayoría de los tallos en la primera clase diamétrica $(72.7 \%$ en BB y $75.2 \%$ en RN) (Figura 3). La distribución por clases de altura de los árboles y arbustos fue similar para ambas estrategias. La cantidad más alta de tallos se 


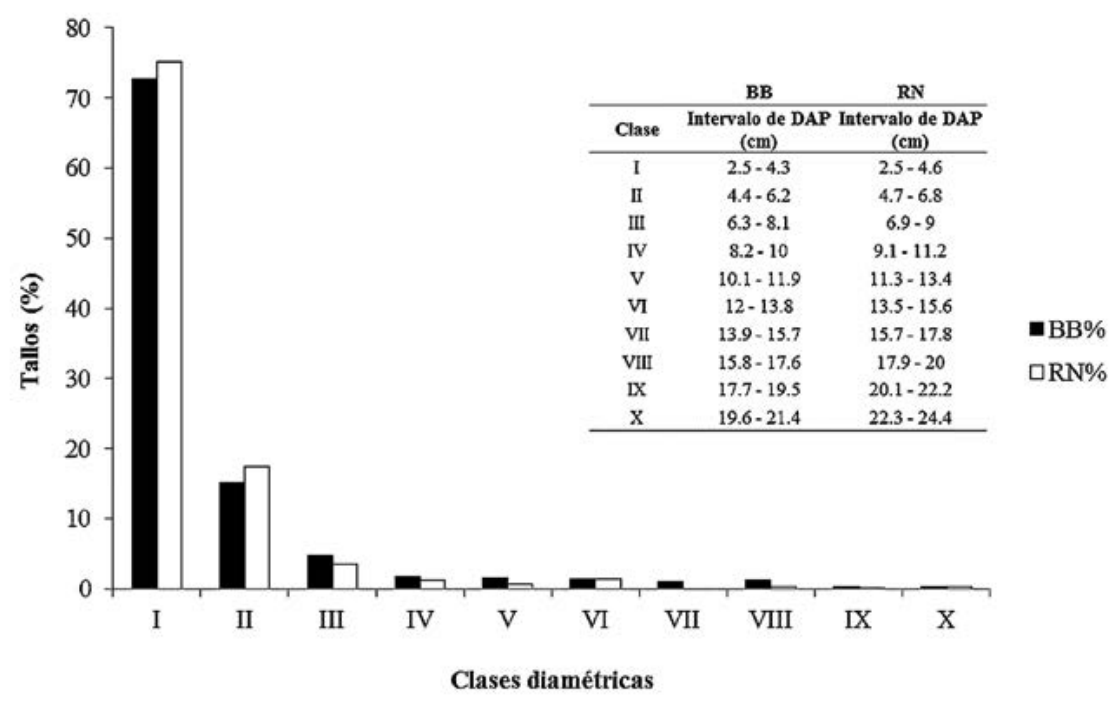

Figura 3. Distribución de árboles y arbustos por clases diamétricas en el bs-T en regeneración del ecoparque Bataclán, Cali, Valle del Cauca, Colombia.

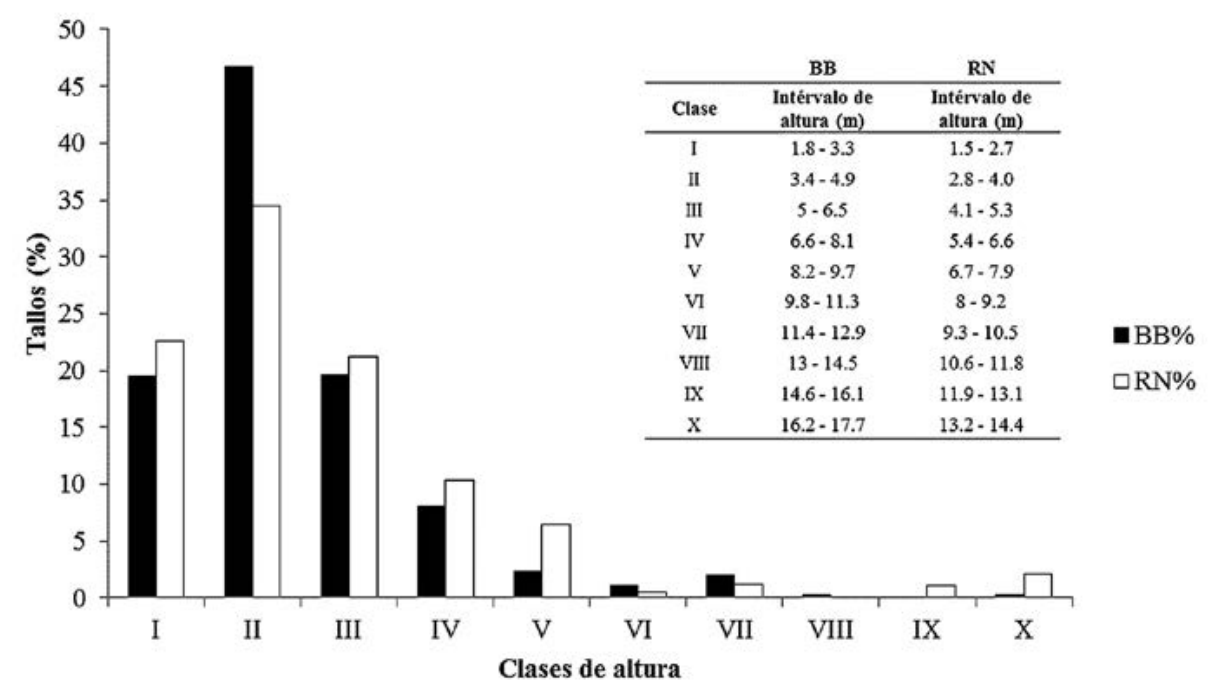

Figura 4. Distribución de árboles y arbustos por clases de altura en el bs-T en regeneración del ecoparque Bataclán, Cali, Valle del Cauca, Colombia.

presentó en la Clase II (46.7 \% en BB y $34.5 \%$ en $\mathrm{RN})$. Asimismo, se observa que para ambas estrategias, la mayoría de clases presentan valores muy similares (Figura 4).

Finalmente, se encontró que la cobertura de hierbas en el suelo está directamente relacionada con el porcentaje de luminosidad que ingresa al sotobosque (coeficiente de Spearman $=0.64133$, $\mathrm{p}<0.001)$. De acuerdo con análisis de especies indicadores, solo la orquídea Catasetum ochraceum Lindl. y el pasto Thrasya petrosa (Trin.) Chase pueden ser consideradas como indicadoras de alta luminosidad (o de sitios abiertos) en el bosque del ecoparque Bataclán $(p<0.05)$ (Tabla 2). 
Tabla 2. Especies herbáceas indicadoras de luminosidad en el bs-T en regeneración del ecoparque Bataclán, Cali, Valle del Cauca, Colombia.

\begin{tabular}{lcccc}
\hline \multicolumn{1}{c}{ Especie } & Luminosidad & Luminosidad (\%) & p & Indicadora \\
\hline Thrasya petrosa & Alta & 72.7 & 0.0012 & $\mathrm{Si}$ \\
Catasetum ochraceum & Alta & 38.4 & 0.0356 & $\mathrm{Si}$ \\
Spermacoce confusa & Alta & 25 & 0.1026 & No \\
Clidemia sericea & Alta & 41.3 & 0.1046 & No \\
Vernonia canescens & Alta & 18.5 & 0.1658 & No \\
Rhynchosia schomburgkii & Alta & 18.3 & 0.1984 & No \\
Scleria bracteata & Media & 47.4 & 0.1266 & No \\
Panicum pilosum & Media & 22 & 0.1456 & No \\
Dioclea sericea & Media & 18.2 & 0.2825 & No \\
Emilia fosbergii & Media & 9.1 & 0.3923 & No \\
Chromolaena laevigata & Baja & 4.3 & 1 & No \\
Calea prunifolia & Baja & 4.3 & 1 & No \\
Mikania banisteriae & Baja & 4.3 & 1 & No \\
\hline
\end{tabular}

\section{DISCUSIÓN}

La composición florística de las dos estrategias de regeneración del bs-T del ecoparque Bataclán presentó una distribución parecida en cuanto a la riqueza de familias y especies de plantas, dentro de cada hábito de crecimiento. En la estrategia BB no se encontraron especies epífitas, mientras que se encontró solamente la especie epífita E. melinanthum en la estrategia RN. La orquídea E. melinanthum es común en los ecosistemas intervenidos y pertenece a una de las mayores familias de plantas epífitas en el Neotrópico (Gentry \& Dodson, 1987). El bajo número de epífitas en el bosque del ecoparque Bataclán se puede explicar por sus condiciones microclimáticas, con alta intensidad lumínica, humedad relativa baja y altas temperaturas, que son factores que afectan la riqueza y distribución de epífitas (Krömer et al., 2007).

La riqueza del bosque del ecoparque Bataclán con 41 especies de plantas se encuentra en el rango registrado por otros trabajos en bs- $\mathrm{T}$, entre $35 \mathrm{y}$
155 especies (Murphy \& Lugo, 1986; IAvH, 1998; Mendoza, 1999; Gillespie et al., 2000; Marulanda et al., 2003; Adarve et al., 2010; Carbonó \& García, 2010; Torres et al., 2012a). Algunas especies como E. biflora, C. minor, Trichanthera gigantea (Humb. \& Bonpl.) Nees, Croton gossypiifolius Vahl, Psidium guajava L., Leucaena leucocephala (Lam.) de Wit y Z. rhoifolium, que fueron encontradas en ambas estrategias de regeneración (a excepción de T. gigantea que solo se encontró en RN y de $P$. guajava y $L$. leucocephala que solo se encontraron en $\mathrm{BB}$ ) han sido reportadas para otros bs-T del Valle del Cauca (González \& Devia, 1994; Adarve et al., 2010; Torres et al., 2012a).

Las familias Anacardiaceae, Myrtaceae y Rutaceae, con mayor IVI en ambas estrategias de regeneración del bosque del ecoparque Bataclán, también han sido registradas como importantes y dominantes para otros bs-T (Gentry, 1995; Ruíz \& Fandiño, 2009; Adarve et al., 2010; Torres et al., 2012a). Aunque Clusiaceae no es una familia dominante en los ecosistemas secos, la especie 
encontrada de esta familia, C. minor, es común en los ecosistemas secos tropicales (Hammel, 2001). El alto nivel de importancia de la familia Myrtaceae, sugiere que el bosque del ecoparque Bataclán se encuentra en regeneración temprana, dado que esta familia se presenta como una de las dominantes en bs-T en regeneración. Para Colombia ha sido reportada en bosques secundarios del Valle del Cauca (Adarve et al., 2010; Torres et al., 2012a) y Norte de Santander (Carrillo et al. 2007).

Por otra parte, el IVI más alto de la familia Melastomataceae se debe principalmente a que es la familia más rica en especies en el bosque estudiado. Esto es excepcional para un bs-T, ya que en general la familia Fabaceae es la que obtiene los mayores valores de IVI en bs-T secundarios en regeneración (Gentry, 1995; IAvH, 1998; Mendoza, 1999; GiIlespie et al., 2000; Marulanda et al., 2003; Adarve et al., 2010; Carbonó \& García, 2010; Torres et al., 2012a). Sin embargo, Vargas (2012), también reporta representantes de esta familia para los bs-T del Valle del Cauca. En este estudio, la familia Fabaceae fue una de las menos representadas (con solo una especie L. leucocephala) y con menor IVI. Esta especie es nativa de México y se ha introducido en el Valle del Cauca y en otras regiones de Colombia como árbol de uso múltiple (e.g. forraje, madera, mejoramiento de pasturas, reforestación, carbono) (Echeverri et al., 1987; Schultze-Kraft, 1994). Otra de las familias típicas de los bs-T según Gentry (1995), que presentó baja riqueza y diversidad de especies en el bosque del ecoparque Bataclán fue Euphorbiaceae. Sin embargo, una de las especies registradas de esta familia en este estudio, Euphorbia tirucalli L., aunque es introducida en América como especie ornamental, es una especie de dosel dominante en los bs-T africanos (Sussman \& Rakotozafy, 1994).

Las familias con mayor riqueza de especies en este bosque (Melastomataceae, Asteraceae y Poaceae), son familias comúnmente encontradas en los bs-T a nivel mundial (Gentry, 1995). Estas, presentan especies de fácil dispersión (e.g. las especies de Asteraceae y Poaceae son dispersadas por el viento), lo que les permite colonizar fácilmente ambientes abandonados. Asimismo, las especies de Melastomataceae son dispersadas por aves y por esto son las primeras especies leñosas en llegar después de una perturbación (Aide et al., 1995).

Las especies que tuvieron los más altos valores de IVI pertenecen a las familias que presentaron mayor IVI. La especie Z. rhoifolium, típicamente dominante en los bs-T de Colombia (González \& Devia, 1994), presentó un IVI alto en la estrategia de BB. Por otra parte, la especie M. prasina, dominante en ambas estrategias de regeneración estuvo ausente solamente en uno de los subcuadrantes de todas las parcelas muestreadas. Esta especie, se ha registrado en Puerto Rico, como dominante en bs-T abandonados entre los doce y los veinticinco años (Aide et al., 1995). Este es el caso del bosque del ecoparque Bataclán, cuyas estrategias de regeneración fueron implementadas hace aproximadamente veinte años.

Los valores del área basal, las clases diamétricas y la altura de las especies leñosas de ambas estrategias de regeneración fueron similares. El área basal (entre $9.2 \mathrm{~m}^{2} \mathrm{ha}^{-1}$ y $9.8 \mathrm{~m}^{2} \mathrm{ha}^{-1}$ ) encontrada para las dos estrategias de regeneración del bosque del ecoparque Bataclán, estuvieron muy por debajo del valor estimado para bs-T que varía entre $17 \mathrm{~m}^{2}$ ha $^{-1}$ y $40 \mathrm{~m}^{2}$ ha $^{-1}$ (Murphy \& Lugo, 1986), lo que indica que el bosque se encuentra en una etapa de sucesión temprana. Asimismo lo sugieren, el patrón de distribución con la mayoría de los tallos en la primera clase diamétrica, en la segunda clase de altura y la altura máxima de los árboles inferior a $18 \mathrm{~m}$ (Adarve et al., 2010).

Para las dos estrategias de regeneración del bosque del ecoparque Bataclán, se encontró que la cobertura de herbáceas está relacionada negativamente con la cobertura del dosel. Las especies $C$. ochraceum y $T$. petrosa fueron indicadores de alta luminosidad y por lo tanto especies indicadoras de baja cobertura vegetal en este bosque.

Las especies trepadoras o escandentes encontradas en ambas estrategias de regeneración del ecoparque Bataclán, pertenecen a las familias 
Fabaceae, Apocynaceae, Aristolochiaceae, Asteraceae y Euphorbiaceae. La ausencia de la familia Bignoniaceae es atípica, ya que se ha registrado como dominante para esta forma de crecimiento en otros bs-T (Gentry, 1995; IAvH, 1998; Mendoza, 1999; Marulanda et al., 2003).

Teniendo en cuenta lo evaluado anteriormente, es posible establecer que la composición y estructura del bosque en regeneración del ecoparque Bataclán no dependen del tipo de estrategia de regeneración, ya que no se encontraron variaciones significativas en ningún parámetro estructural o florístico al comparar las dos estrategias. En consecuencia, las barreras de $P$. aurea después de veinte años de establecidas, no incidieron ni en la diversidad, ni en la composición de especies de plantas establecidas en el bosque. Por otro lado, los factores que regulan la riqueza y diversidad de especies en este bosque (e.g. Iluvias, fertilidad del suelo, tasa de recambio) se encuentran más relacionados con las condiciones de la zona en general (Givnish, 1999), que con las características de cada estrategia de regeneración desarrollada en el bosque.

Las características del bosque, con una elevada proporción de especies pioneras, de fácil dispersión y rápido crecimiento y una baja similitud a nivel florístico con otros bosques secos, se deben principalmente a los fuertes niveles de disturbio por la acción humana (Carrillo et al., 2007). Para el caso de este bosque la continua amenaza de incendios forestales para invadir la zona es el mayor disturbio antrópico. Adicionalmente, el bosque no se encuentra cerca de un recurso que sea fuente de especies de bs-T. Así, la zona ha recibido especies dispersadas por aves como lo son las especies de Melastomataceae y Myrtaceae con frutos en baya y de Clusiaceae con frutos en cápsula con semillas que exponen un arilo (Gentry \& Dodson, 1987). Otra de las fuentes de especies más continuas para la vegetación del ecoparque Bataclán, es la siembra de especies de árboles realizada por el DAGMA y los habitantes del sector alrededor de sus casas (L. A. Forero, com. pers.). Debido a esto, el bosque del ecoparque Bataclán, consiste de una mezcla de especies plantadas y otras naturalmente regeneradas.

Según Janzen (1988), cuando un área que antes fue bs-T es dejada para su regeneración es muy posible que siga por uno de dos caminos, por un lado, si está lejos de un bs-T aledaño, será colonizada por plantas que sean fácilmente dispersadas por el viento y ya que estas especies no son muy hospitalarias con los animales, el bosque permanecerá dominado por especies con este tipo de dispersión. O por otro lado, si en la zona hay alguna atracción para que las aves perchen cerca, ocurrirá el establecimiento de plantas que atraen más dispersores, lo que hará de esta un área con mayor riqueza, pero igual de artificial que la formada por especies dispersadas por el viento. La comunidad vegetal del ecoparque Bataclán, muy posiblemente es el resultado de ambos procesos, ya que la mayoría de las especies de árboles y arbustos de la zona son dispersadas por aves y la mayoría de especies de hierbas, trepadoras y epífitas, son dispersadas por el viento. Otro de los factores importantes que ha regulado la composición del bosque ha sido la siembra de especies por parte del DAGMA y la comunidad de la zona.

Debido a la larga historia de disturbio que ha presentado la comunidad vegetal de la zona, no es posible conocer las características de la vegetación inicial. Es importante resaltar que el bosque del ecoparque Bataclán se encuentra muy alejado de otros parches de bs-T y, por esta razón, es posible que la comunidad vegetal se encuentre aislada de una fuente de especies típicas de esta zona de vida, lo que explicaría el porqué pese a que tiene las características climáticas de un bs-T su composición no es tan representativa de este ecosistema. De hecho, la vegetación encontrada para este bosque es similar a la registrada para otras zonas montañosas cercanas al ecoparque Bataclán (Gamboa, 1995; Botina \& García, 2005). Lo anterior indica que la comunidad vegetal del ecoparque Bataclán se encuentra representada por especies pioneras provenientes de bs-T y de otras zonas de vida aledañas al bosque. 


\section{CONCLUSIONES Y RECOMENDACIONES}

Se concluye que la composición y estructura de la comunidad vegetal no difiere entre las dos estrategias de regeneración, lo que indica que el manejo dado a la vegetación en la zona es insuficiente para causar un impacto positivo en la sucesión. También es posible concluir que los factores que regulan la riqueza y diversidad de especies en este bosque se encuentran más relacionados con las condiciones climáticas, geográficas, edáficas y por la intervención antrópica que ocurre en la zona en general (que son las que regulan el establecimiento de las especies), que con las características de cada estrategia de regeneración implementada.

Adicionalmente, por las características estructurales del bosque del ecoparque Bataclán en ambas estrategias de regeneración (i.e. altura, área basal, DAP) se concluye que la vegetación se encuentra en un estado sucesional temprano, que está dominado por especies de fácil dispersión. Debido a que el bosque del ecoparque Bataclán se encuentra lejos de otros parches de bs-T del Valle del Cauca, este no comparte muchas especies típicas del bosque seco. Así, el bosque el ecoparque Bataclán es una mezcla de especies plantadas y naturalmente regeneradas en la zona.

Como recomendación general, con el objetivo de recuperar la cobertura vegetal de la zona y detener el proceso de erosión de la misma, es necesario diseñar una estrategia de restauración con especies representativas de la zona de vida en que se encuentra el ecoparque Bataclán, es decir de bs-T, para que logren establecerse efectivamente en el bs-T del ecoparque Bataclán.

\section{AGRADECIMIENTOS}

A la bióloga Linda Mariana Cárdenas Henao por su asesoría, apoyo y acompañamiento en cada parte de esta investigación. A Luz Ángela Forero, del Departamento Administrativo de Gestión del Medio Ambiente (DAGMA) por la gestión en el financiamiento de este proyecto. A Cesar Luna y Don José, personal del ecoparque Bataclán, por su acompañamiento y apoyo en las labores de campo. Al profesor Philip Silverstone Sopkin, director del herbario CUVC, y su personal de trabajo. A la Universidad del Valle, Departamento de Biología, sección de Botánica. Al biólogo Edier Alberto Soto Medina, por su apoyo en el análisis estadístico. A los biólogos: Ángela María González Colorado, Martín Llano Almario, Johan Kelber Home Ramírez y Rodrigo Lozano, por su colaboración en el trabajo de campo. A Jaime Bernal Hadad, por la ayuda en la búsqueda bibliográfica.

\section{REFERENCIAS BIBLIOGRÁFICAS}

Adarve, J. B., Torres, A. M., Home, J., Vargas, J. A., Rivera, K., Duque, O. L., Cárdenas, M., Londoño, V. \& González A. M. (2010). Estructura y riqueza florística del Parque Natural Regional el Vínculo-Buga, Colombia. Cespedesia, 32(90/91), 23-38.

Aide, T. M., Zimmerman, J. K., Herrera, L., Rosario, M. \& Serrano, M. (1995). Forest recovery in abandoned tropical pastures in Puerto Rico. Forest Ecology and Management, 77, 77-86.

Botina, J. R. \& García, L. (2005). Árboles y arbustos del ecoparque río Pance. Cali: Corporación Autónoma Regional del Valle del Cauca (CVC). 72 p.

Brown, S. \& Lugo, A. E. (1990). Tropical secondary forest. Journal of Tropical Ecology, 6(1), 1-32.

Calle, Z. (1994). Diversidad biológica y diálogo de saberes, memorias del curso de campo sobre biodiversidad y recursos genéticos indígenas y campesinos. $1^{\text {a }}$ ed. Cali: Maestría en desarrollo sostenible de sistemas agrarios. $142 \mathrm{p}$.

Camacho, J. E., Ortiz, R., Walschburger, T. \& Hurtado, A. (1992). Estado de la biodiversidad en Colombia. En G. Halffter (ed.). La diversidad biológica de Iberoamérica I (pp. 41-153). México: Instituto de Ecología, A. C.

Carbonó, E. \& García, H. (2010). La vegetación terrestre en la ensenada de Neguanje, Parque Nacional Natural Tayrona (Magadalena, Colombia). Caldasia, $32(2), 235-256$. 
Carim, S., Schwarzt, G. \& Fernandes, M. (2007). Riqueza de espécies, estrutura e composição florística de uma floresta secundária de 40 años no leste da Amazônia. Acta Botânica Brasilica, 21(2), 293-308.

Carrillo, M., Rivera, O. \& Sánchez, R. (2007). Caracterización florística y estructural del bosque seco tropical del cerro Tasajero, San José de Cúcuta (Norte de Santander), Colombia. Actualidades Biológicas, 29(86), 55-73.

DAGMA (Departamento Administrativo de Gestión de Medio Ambiente). (2009). Informe anual de los recursos naturales y del medio ambiente del departamento administrativo de gestión del medio ambiente vigencia 2009. Cali: Alcaldía de Santiago de Cali. 72 p.

DAGMA (Departamento Administrativo de Gestión de Medio Ambiente). (2010). Ecoparques urbanos de Cali, un espacio de vida. Cali: Feriva. 34 p.

Echeverri, J. D., Gómez-Carabalí, A., Pizarro, E. A. \& Franco, L. H. (1987). Evaluación agronómica de accesiones de Leucaena en el Valle del Cauca, Colombia. Pasturas Tropicales, 9(3), 25-29.

EstimateS. Colwell, R. K. (2013). EstimateS: Statistical estimation of species richness and shared species from samples. Version 9.1. Storrs: University of Connecticut. Recuperado de http://purl.oclc.org/estimates

Forero, L. A. Funcionaria del Departamento Administrativo de Gestión de Medio Ambiente (DAGMA), Cali, Valle del Cauca, Colombia. Comunicación personal (2011).

Font-Quer, P. (1953). Diccionario de Botánica. Madrid: Ediciones Península. 1244 p.

Gamboa, M. A. (1995). Contribuciones a la Flora de los Farallones de Cali. I. Vereda Pico de Águila. Trabajo de grado de pregrado. Cali: Universidad del Valle, Facultad de Ciencias. 316 p.

Gentry, A. H. (1995). Diversity and floristic composition of neotropical dry forests. En S. H. Bullock, Mooney, H. A. \& Medina, E. (eds.). Seasonally dry tropical forest (pp. 146-194). Edimburg: Cambridge University Press.

Gentry, A. \& Dodson, C.H. (1987). Diversity and biogeography of Neotropical vascular epiphytes. Annals of Missouri Botanical Garden, 74(2), 205-233.
Gillespie, T. W., Grijalva, A. \& Farris, C. N. (2000). Diversity, composition, and structure of tropical dry forest in Central America. Plant Ecology, 147(1), 37-47.

Givnish, T. J. (1999). On the causes of gradients in tropical tree diversity. Journal of Ecology, 87(2), 193-210.

González, S. M. \& Devia, W. (1994). Caracterización fisionómica de la flora de un bosque seco secundario en el corregimiento de Mateguadua, Tuluá VaIle. Cespedesia, 20(66), 35-65.

Hammel, B. E. (2001). Clusia. En W. D. Stevens, C. Ulloa-Ulloa, C., Pool, A. \& Montiel, O. M. (eds.). Flora De Nicaragua: Introducción a Gimnospermas y Angiospermas (Acanthaceae-Euphorbiaceae) (pp. 620-625). Saint Louis: Missouri Botanical Garden Press.

Hammer, Ø., Harper, D. A. T. \& Ryan, P. D. (2001). PAST: Paleontological Statistics software package for education and data analysis, Versión 2.17c. Palaeontologia Electronica, 4(1), 9.

Holdridge, L. R. (1967). Life zone ecology. San José de Costa Rica: Tropical Science Center. 149 p.

IAvH (Instituto Alexander Von Humboldt). (1998). El bosque seco tropical (bs-T) en Colombia. Bogotá: Programa de Inventario de la Biodiversidad Grupo de Exploraciones y Monitoreo Ambiental GEMA. $24 \mathrm{p}$.

IDEAM (Instituto de Hidrología, Meteorología y Estudios Ambientales de Colombia). (2005). Atlas Climatológico de Colombia. Bogotá: Imprenta Nacional de Colombia. 217 p.

Janzen, D. H. (1988). Management of habitat fragments in a tropical dry forest: Growth. Annals of Missouri Botanical Garden, 75(1), 105-116.

Jørgensen, P. M., Ulloa-Ulloa, C., León, B., León-Yánez, S., Beck, S. G., Nee, M., Zarucchi, J. L., Celis, M., Bernal, R. \& Gradstein, R. (2011). Regional patterns of vascular plant diversity and endemism. En Herzog, S. K., Martínez, R., Jørgensen, P. M. (eds.). Climate Change and Biodiversity in the Andes (pp. 192-203). São José dos Campos: JAI and SCOPE.

Krömer, T., Kessler, M. \& Gradstein, S. R. (2007). Vertical stratification of vascular epiphytes in submontane and montane forest of the Bolivian Andes: 
the importance of the understory. Plant Ecology, 189(2), 261-278.

Londoño, X. (2004). Bambúes exóticos en Colombia. Armenia: Sociedad Colombiana del Bambú. 74 p.

Marulanda, L. O., Uribe, A., Velásquez, P., Montoya, M. A., Idárraga, A., López, M. C. \& López, J. M. (2003). Estructura y composición de la vegetación de un fragmento de bosque seco en San Sebastián, Magdalena (Colombia). I. Composición de plantas vasculares. Actualidades Biológicas, 25(78), 17-30.

Mendoza, H. (1999). Estructura y riqueza florística del bosque seco tropical en la región Caribe y el valle del río Magdalena, Colombia. Caldasia, 21(1), 70-94.

Murphy, P. G. \& Lugo A. E. (1986). Ecology of tropical dry forest. Annual Review of Ecology and Systematics, 17, 67-88.

PC-ORD. McCune, B. \& Mefford, M. J. (1999). PC-ORD Multivariate analysis of ecological data, Version 4.0. Gleneden Beach: MjM Software. Recuperado de http://www.pcord.com/pcordwin.htm

Rangel, J. O. (2011). Colombia diversidad biótica XI, patrones de la estructura y de la riqueza de la vegetación en Colombia. Bogotá: Instituto de Ciencias Naturales. 486 p.

Rangel, J. O. \& Velázquez, A. (1997). Métodos de estudio de la vegetación. En J. O. Rangel, Lowy, P. D. \& Aguilar, M. (eds.). Colombia diversidad biótica II (pp. 59-87). Bogotá: Editorial Guadalupe Ltda.

Rodríguez, F. \& Arrroyo, J. (2004). La necesidad de la experimentación en ecología y conservación: una aproximación para la restauración forestal en el Parque Natural los Alcornocales. Almoraima, 3, 137-144.

Rudell, T. K., Bates, D. \& Machinguiashi, R. (2002). A tropical forest transition? agricultural change, out-migration, and secondary forest in the Ecuadorian Amazon. Annals of the Association of American Geographers, 92(1), 87-102.

Ruíz, J. \& Fadiño, M. C. (2009). Estado del bosque seco tropical e importancia relativa de su flora leñosa, islas de la Vieja Providencia y Santa Catalina, Colombia, Caribe suroccidental. Revista de la Academia Colombiana de Ciencias, 33(126), 5-15.
Sánchez, G. (2002). Desarrollo y medio ambiente: una mirada a Colombia. Economía y Desarrollo, 1(1), 79-98.

Schultze-Kraft, R. (1994). El "psyllid" de Leucaena también puede ser un problema en América tropical. Pasturas Tropicales, 16(2), 48-50.

Silverstone-Sopkin, P. A. Docente de la Universidad del Valle, Cali, Valle del Cauca, Colombia. Comunicación personal (2011).

Sussman, R. W. \& Rakotozafy, A. (1994). Plant diversity and structural analysis of a tropical dry forest in southwestern Madagascar. Biotropica, 26(3), 241-254.

Torres, A. M., Adarve, J. B., Cárdenas, M., Vargas, J. A., Londoño, V., Rivera, K., Home, J., Duque, O. L. \& González, A. M. (2012a). Dinámica sucesional de un fragmento de bosque seco tropical del Valle del Cauca, Colombia. Biota Colombiana, 13(2), 66-87

Torres, A. M., E. J. Peña, O. Zúñiga \& J. A. Peña. (2012b). Evaluación del impacto de actividades antrópicas en el almacenamiento de carbono en biomasa vegetal en ecosistemas de alta montaña de Colombia. Boletín Científico Museo de Historia Natural, 16(1), 132-142.

Uslar, Y. V., Mostacedo, B. \& Saldias, M. (2004). Composición, estructura y dinámica de un bosque seco semideciduo en Santa Cruz, Bolivia. Ecología en Bolivia, 39(1), 25-43.

Vallejo, M. I., Londoño, A. C., López, R., Galeano, G., Álvarez, E. \& Devia, W. (2005). Establecimiento de parcelas permanentes en bosques de Colombia. Bogotá: Instituto de Investigación de Recursos Biológicos Alexander von Humboldt. 310 p.

Van Der Hammen, T. \& Rangel J. O. (1997). El estudio de la vegetación en Colombia (Recuento histórico-tareas futuras). En J. O. Rangel, Lowy, P. D. \& Aguilar, M. (eds.). Colombia diversidad biótica II (pp. 17-57). Bogotá: Editorial Guadalupe Ltda.

Vargas, W. (2012). Los bosques secos del Valle del Cauca, Colombia: una aproximación a su flora actual. Biota Colombiana, 13(2), 102-164.

Velásquez, A. \& Meyer, H. (1994). Ofertas y amenazas ambientales en Cali. $2^{\text {a }}$ ed. Cali: Publicaciones ocasionales del OSSO, № 3.37 p. 


\section{Anexos}

\section{Anexo A. Composición florística por hábito de crecimiento en las dos estrategias de regeneración del bosque del ecoparque Bataclán, Cali, Valle del Cauca, Colombia.}

\begin{tabular}{|c|c|c|c|c|c|}
\hline $\begin{array}{l}\text { Hábito de } \\
\text { crecimiento }\end{array}$ & Familia & Especie & BB & RN & No. Herbario CUVC \\
\hline \multirow{23}{*}{$\begin{array}{l}\text { Árboles y } \\
\text { arbustos }\end{array}$} & Acanthaceae & Trichanthera gigantea (Humb. \& Bonpl.) Nees & 0 & 1 & --- \\
\hline & Anacardiaceae & Mangifera indica L. & 1 & 1 & 47824 \\
\hline & Clusiaceae & Clusia minor L. & 1 & 1 & $50019,50018,47823$ \\
\hline & \multirow{2}{*}{ Euphorbiaceae } & Croton gossypiifolius Vahl & 1 & 0 & 47828 \\
\hline & & Euphorbia tirucalli L. & 1 & 0 & 50048,50012 \\
\hline & Fabaceae & Leucaena leucocephala (Lam.) de Wit & 1 & 0 & --- \\
\hline & \multirow{2}{*}{ Lauraceae } & Cinnamomum triplinerve (Ruiz \& Pav.) Kosterm. & 1 & 1 & 50017 \\
\hline & & Persea americana Mill. & 1 & 0 & --- \\
\hline & \multirow{3}{*}{ Malvaceae } & Pachira speciosa Triana \& Planch. & 0 & 1 & --- \\
\hline & & Henriettella seemannii Naudin & 1 & 1 & 50013,47833 \\
\hline & & Miconia albicans (Sw.) Steud. & 1 & 1 & 50035 \\
\hline & \multirow[t]{3}{*}{ Melastomataceae } & Miconia minutiflora (Bonpl.) DC. & 1 & 1 & 50030 \\
\hline & & Miconia prasina (Sw.) DC. & 1 & 1 & $50015,50014,47825$ \\
\hline & & Miconia rubiginosa (Bonpl.) DC. & 1 & 1 & 50038 \\
\hline & Moraceae & Ficus elastica Roxb. ex Hornem. & 1 & 0 & --- \\
\hline & \multirow[t]{2}{*}{ Myrsinaceae } & Myrsine guianensis (Aubl.) Kuntze & 1 & 1 & 50043,47826 \\
\hline & & Eucalyptus microcorys F.Muell. & 0 & 1 & 50047 \\
\hline & \multirow[t]{2}{*}{ Myrtaceae } & Eugenia biflora (L.) DC. & 1 & 1 & 50016,47827 \\
\hline & & Psidium guajava L. & 1 & 0 & --- \\
\hline & Oleaceae & Fraxinus chinensis Roxb. & 1 & 0 & --- \\
\hline & \multirow{2}{*}{ Poaceae } & Bambusa vulgaris Schrad. & 1 & 0 & --- \\
\hline & & Phyllostachys aurea Rivière \& C.Rivière & 1 & 0 & --- \\
\hline & \multirow{3}{*}{ Rutaceae } & Citrus limon (L.) Osbeck & 1 & 1 & --- \\
\hline & & Zanthoxylum rhoifolium Lam. & 1 & 1 & 47414 \\
\hline \multirow{10}{*}{ Hierbas } & & Chromolaena laevigata (Lam.) R.M.King \& H.Rob. & 1 & 0 & 50041 \\
\hline & \multirow[t]{2}{*}{ Asteraceae } & Calea prunifolia Kunth & 1 & 0 & 50044 \\
\hline & & Emilia fosbergii Nicolson & 0 & 1 & 50037 \\
\hline & Cyperaceae & Scleria bracteata Cav. & 1 & 1 & 50021 \\
\hline & Fabaceae & Dioclea sericea Kunth* & 1 & 1 & 50022 \\
\hline & Melastomataceae & Clidemia sericea D. Don & 1 & 1 & 50020 \\
\hline & Orchidaceae & Catasetum ochraceum Lindl. & 0 & 1 & 50033 \\
\hline & \multirow{2}{*}{ Poaceae } & Panicum pilosum Sw. & 1 & 1 & 50039 \\
\hline & & Thrasya petrosa (Trin.) Chase & 0 & 1 & 50031 \\
\hline & Rubiaceae & Spermacoce tenuior $\mathrm{L}$. & 0 & 1 & 50029 \\
\hline \multirow{6}{*}{ Trepadoras } & Apocynaceae & Mandevilla bracteata (Kunth) Kuntze & 1 & 1 & 50028 \\
\hline & Aristolochiaceae & Aristolochia ringens Vahl & 1 & 1 & 50027 \\
\hline & \multirow[t]{2}{*}{ Asteraceae } & Vernonia canescens Kunth* & 1 & 1 & 50046 \\
\hline & & Mikania banisteriae DC.* & 0 & 1 & 50040 \\
\hline & Euphorbiaceae & Dalechampia scandens L. & 1 & 0 & 50026 \\
\hline & Fabaceae & Rhynchosia schomburgkii Benth.* & 1 & 1 & $50025,50024,50023$ \\
\hline Epífitas & Orchidaceae & Epidendrum melinanthum Schltr. & 0 & 1 & 50036 \\
\hline
\end{tabular}

BB: Regeneración Natural con Barreras de Bambú, RN: Regeneración Natural sin Barreras de Bambú. *Especies que pueden ser encontradas con hábito herbáceo o escandente o trepador. 
Anexo B. Características estructurales de los árboles y arbustos registrados en dos estrategias de regeneración del bosque del ecoparque Bataclán, Cali, Valle del Cauca, Colombia.

\begin{tabular}{lcccccccc}
\hline \multirow{2}{*}{ Especie } & \multicolumn{2}{c}{ Abundancia (Tallos) } & \multicolumn{2}{c}{ Frecuencia } & \multicolumn{2}{c}{ Área basal $\left(\mathbf{m}^{2}\right)$} & \multicolumn{2}{c}{ IVI (\%) } \\
\cline { 2 - 9 } & $\mathbf{B B}$ & $\mathbf{R N}$ & $\mathbf{B B}$ & $\mathbf{R N}$ & $\mathbf{B B}$ & $\mathbf{R N}$ & $\mathbf{B B}$ & $\mathbf{R N}$ \\
\hline Bambusa vulgaris & 1 & $\mathrm{~N}$ & 1 & $\mathrm{~N}$ & 0.003 & $\mathrm{~N}$ & 1.4 & $\mathrm{~N}$ \\
Cinnamomum triplinerve & 21 & 8 & 7 & 5 & 0.068 & 0.08 & 15.5 & 12.8 \\
Citrus limon & 2 & 1 & 2 & 1 & 0.002 & 0 & 2.6 & 1.5 \\
Clusia minor & 38 & 168 & 11 & 15 & 0.068 & 0.23 & 22.3 & 56.5 \\
Croton gossypiifolius & 9 & $\mathrm{~N}$ & 3 & $\mathrm{~N}$ & 0.014 & $\mathrm{~N}$ & 5.5 & $\mathrm{~N}$ \\
Eucalyptus microcorys & $\mathrm{N}$ & 1 & $\mathrm{~N}$ & 1 & $\mathrm{~N}$ & 0.05 & $\mathrm{~N}$ & 4.6 \\
Eugenia biflora & 85 & 71 & 12 & 11 & 0.091 & 0.07 & 32.3 & 28.3 \\
Euphorbia tirucalli & 1 & $\mathrm{~N}$ & 1 & $\mathrm{~N}$ & 0.006 & $\mathrm{~N}$ & 1.6 & $\mathrm{~N}$ \\
Ficus elastica & 1 & $\mathrm{~N}$ & 1 & $\mathrm{~N}$ & 0.033 & $\mathrm{~N}$ & 3.6 & $\mathrm{~N}$ \\
Fraxinus chinensis & 2 & $\mathrm{~N}$ & 2 & $\mathrm{~N}$ & 0.003 & $\mathrm{~N}$ & 2.6 & $\mathrm{~N}$ \\
Henriettella seemannii & 28 & 5 & 6 & 4 & 0.037 & 0.01 & 13.3 & 6.2 \\
Leucaena leucocephala & 1 & $\mathrm{~N}$ & 1 & $\mathrm{~N}$ & 0.001 & $\mathrm{~N}$ & 1.3 & $\mathrm{~N}$ \\
Mangifera indica & 42 & 15 & 6 & 5 & 0.379 & 0.19 & 40.2 & 21.1 \\
Miconia albicans & 3 & 1 & 1 & 1 & 0.005 & 0 & 1.9 & 1.4 \\
Miconia minutiflora & 6 & 4 & 3 & 2 & 0.009 & 0 & 4.7 & 3.3 \\
Miconia prasina & 328 & 294 & 14 & 15 & 0.298 & 0.39 & 87.3 & 83.8 \\
Miconia rubiginosa & 28 & 182 & 7 & 13 & 0.057 & 0.32 & 15.8 & 62 \\
Myrsine guianensis & 2 & 1 & 2 & 1 & 0.003 & 0 & 2.6 & 1.4 \\
Pachira speciosa & $\mathrm{N}$ & 1 & $\mathrm{~N}$ & 1 & $\mathrm{~N}$ & 0 & $\mathrm{~N}$ & 1.6 \\
Persea americana & 3 & $\mathrm{~N}$ & 1 & $\mathrm{~N}$ & 0.023 & $\mathrm{~N}$ & 3.1 & $\mathrm{~N}$ \\
Phyllostachys aurea & 2 & $\mathrm{~N}$ & 2 & $\mathrm{~N}$ & 0.001 & $\mathrm{~N}$ & 2.5 & $\mathrm{~N}$ \\
Psidium guajava & 7 & $\mathrm{~N}$ & 3 & $\mathrm{~N}$ & 0.02 & $\mathrm{~N}$ & 5.7 & $\mathrm{~N}$ \\
Trichanthera gigantea & $\mathrm{N}$ & 2 & $\mathrm{~N}$ & 1 & $\mathrm{~N}$ & 0 & $\mathrm{~N}$ & 1.8 \\
Zanthoxylum rhoifolium & 32 & 7 & 10 & 3 & 0.261 & 0.13 & 34.3 & 13.9 \\
Total & 642 & 761 & 96 & 79 & 1.38 & 1.47 & 300 & 300 \\
\hline
\end{tabular}

N: especie no registrada. BB: Regeneración Natural con Barreras de Bambú. RN: Regeneración Natural sin Barreras de Bambú.

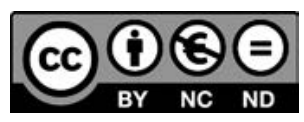


